https://doi.org/10.15407/ujpe65.5.428

M.P. KOZLOVSKII, O.A. DOBUSH

Institute for Condensed Matter Physics, Nat. Acad. of Sci. of Ukraine

(1, Svientsitsky Str., Lviv 79011, Ukraine)

\title{
PHASE BEHAVIOR OF A CELL FLUID MODEL WITH MODIFIED MORSE POTENTIAL
}

\begin{abstract}
The present article gives a theoretical description of a first-order phase transition in the cell fluid model with a modified Morse potential and an additional repulsive interaction. In the framework of the grand canonical ensemble, the equation of state of the system in terms of chemical potential-temperature and terms of density-temperature is calculated for a wide range of the density and temperature. The behavior of the chemical potential as a function of the temperature and density is investigated. The maximum and minimum admissible values of the chemical potential, which approach each other with decreasing the temperature, are exhibited. The existence of a liquid-gas phase transition in a limited temperature range below the critical $T_{\mathrm{c}}$ is established.

Ke ywords: cell fluid model, coexistence curve, collective variables, equation of state, firstorder phase transition.
\end{abstract}

\section{Introduction}

Construction of the equation of state is a topical problem of studying the phase behavior of a system of interacting particles. Nowadays, several such equations are, to a great extent, phenomenological in nature and well describe the properties of real substances. There are few approaches in which such equations were obtained at the level of microscopic description. Among them are methods based on investigating the behavior of the virial equation of state $[1,2]$. The latter describes the behavior of systems at the thermodynamic boundary. Nevertheless, the consideration of higher viral coefficients causes problems in displaying the liquid branch of the phase diagram [3]. Another well-known approach to describe the phase behavior of fluids is the theory of integral equations. This method is mostly a numerical procedure for the structural analysis of a system based on the calculation of paired correlation functions. One of its implementations is the self-consistent Ornstein-Zernike approximation (SCOZA) $[4,5]$.

Previously [6-8], we proposed a cell fluid model applied to describe a first-order phase transition using different types of interaction potentials. Particularly in [6], the grand partition function was calculated, and the equation of state of a system with the CurieWeiss potential was obtained. Without using any ap-

(C) M.P. KOZLOVSKII, O.A. DOBUSH, 2020 proximations, we established that such system possesses a cascade of first-order phase transitions describing a sequence of phases with increasing density. The emergence of multiple phase transitions, rather than a single one, was associated with particles being point-like. The explanation of the appearance of high-density phases in this model is such that a term forbidding the excessive concentration of particles in a cell is absent in the interaction potential. Usually, while describing the fluid systems, the hard-sphere (or soft-sphere) potential limiting the maximum density is used $[9,10]$. In the present paper, a supplemental term of soft repulsion is included in the interaction potential, and, afterward, an appropriate equation of state is calculated. For this purpose, a modified Morse potential is used, which, along with the attractive and repelling components, contains a part describing the additional soft-wall repulsion. The inclusion of such a term in the interaction potential enables us to specify a distinct reference system, which makes it possible to calculate the Jacobian of the transition from density variables to collective variables. The set of collective variables is natural for describing the collective effects, including phase transitions.

The novelty of this work consists in the extension of the previously obtained results [11] over a wide temperature range (including the temperature at which the liquid-gas phase transition region ends). This is

ISSN 2071-0194. Ukr. J. Phys. 2020. Vol. 65, No. 5 
made possible by the use of a modified interaction potential, which contains the additional soft repulsion.

\section{A Model}

Consider a system of $N$ interacting particles located in the volume $V$. The potential of interaction is a function of the distance $r$ between particles

$$
\begin{aligned}
& U(r)=C_{H}\left\{A e^{-n_{0}\left(r-R_{0}\right) / \alpha}+e^{-\gamma\left(r-R_{0}\right) / \alpha}-\right. \\
& \left.-2 e^{-\left(r-R_{0}\right) / \alpha}\right\} .
\end{aligned}
$$

In the last expression, $R_{0}$ is the equilibrium distance between two particles (location of the minima of the potential), $\alpha$ is the effective radius of interaction, $\gamma, n_{0}$ are some parameters of the model, $C_{H}$ and $A$ are the normalization constants. The location of the minima of the function $U(r)$ at $r=R_{0}$ can be found from the condition

$\left.\frac{\partial U(r)}{\partial r}\right|_{R_{0}}=0 \Rightarrow A=\frac{2-\gamma}{n_{0}}$.

The depth of the potential well, which, according to [13], is equal to the energy of dissociation $D$

$U\left(R_{0}\right)=-D$.

The value of the constant $C_{H}$ is determined from condition (3)

$C_{H}=D \frac{n_{0}}{n_{0}+\gamma-2}$.

Obviously, the interaction potential (1) has two parameters $n_{0}$ and $\gamma$, since the characteristic quantities $R_{0}, D$, and $\alpha$ of a specific physical system are known from numerical computations and their comparison with experiments. In particular, for sodium (Na) [13], we have

$R_{0}=5.3678 \AA, \quad \frac{1}{\alpha}=0.5504 \AA^{-1}, \quad \frac{R_{0}}{\alpha}=2.9544$.

The constant of dissociation energy $D$ for Na takes on a value [14]

$D=0.9241 \times 10^{-13}$ ergs.

Note that, in the case of $n_{0} \gg 1$, the former term in (1) corresponds to the hard core (it goes to infinity for any $r<R_{0}$ and turns to zero for $r>R_{0}$ ). The case of $C_{H} \rightarrow D$ as $A \rightarrow 0$ yields the ordinary Morse potential [13].

To write a lattice form of the Morse potential (1), we use the following notations:

$$
\begin{aligned}
& \tilde{\Phi}^{(r)}(r)=C_{H} e^{-\gamma\left(r-R_{0}\right) / \alpha}, \\
& \tilde{\Phi}^{(a)}(r)=2 C_{H} e^{-\left(r-R_{0}\right) / \alpha}, \\
& \tilde{\Psi}(r)=C_{H} A e^{-n_{0}\left(r-R_{0}\right) / \alpha} .
\end{aligned}
$$

Then we define $l_{12}=\left|\mathbf{l}_{1}-\mathbf{l}_{2}\right|$ as the distance between two cells $l_{1}$ and $l_{2}$. According to [6], the volume of the system is conditionally divided into $N_{v}$ cubic cells such that $V=c^{3} \cdot N_{v}$, where $c$ is the side, and $v=c^{3}$ is the volume of each cell. The set of cell vectors is defined as

$\Lambda=\left\{l=\left(l_{x}, l_{y}, l_{z}\right) \mid l_{i}=c \cdot n_{i} ; n_{i}=1,2, \ldots, N_{i} ;\right.$

$\left.i=x, y, z ; N_{i}=N_{v}^{1 / 3}\right\}$.

In the thermodynamic limit as $V \rightarrow \infty, N_{v} \rightarrow \infty$, and $v=V / N_{v}=$ const, we have

$\tilde{\Phi}_{l_{12}}^{(r)}=C_{H} e^{-\gamma\left(l_{12}-c\right) / \alpha_{R} c}$,

$\tilde{\Phi}_{l_{12}}^{(a)}=2 C_{H} e^{-\left(l_{12}-c\right) / \alpha_{R} c}$,

$\tilde{\Psi}_{l_{12}}=C_{H} A e^{-n_{0}\left(l_{12}-c\right) / \alpha_{R} c}$.

Here, $\alpha_{R}=\alpha / R_{0}$ is a dimensionless quantity. Let $l_{12}=x c$. Then expressions (8) take the following form:

$\tilde{\Phi}^{(r)}(x)=C_{H} e^{-\gamma(x-1) / \alpha_{R}}$,
$\tilde{\Phi}^{(a)}(x)=2 C_{H} e^{-(x-1) / \alpha_{R}}$,
$\tilde{\Psi}(x)=C_{H} A e^{-n_{0}(x-1) / \alpha_{R}}$.

The latter expressions coincide with (6), if one takes into account that $r=x R_{0}, \alpha_{R}=\alpha / R_{0}$. The Fourier transforms of the interaction potentials (8) are as follows:

$$
\begin{aligned}
& \Phi^{(r)}(k)=C_{H} 8 \pi e^{\gamma / \alpha}\left(\frac{\alpha}{\gamma}\right)^{3}\left(1+\left(\frac{\alpha c}{\gamma}\right)^{2} k^{2}\right)^{-2} \\
& \Phi^{(a)}(k)=C_{H} 16 \pi e^{1 / \alpha} \alpha^{3}\left(1+(\alpha c k)^{2}\right)^{-2} \\
& \Psi(k)=C_{H} A 8 \pi e^{n_{0} / \alpha}\left(\frac{\alpha}{n_{0}}\right)^{3}\left(1+\left(\frac{\alpha c}{n_{0}}\right)^{2} k^{2}\right)^{-2}
\end{aligned}
$$


To simplify notations, let $\alpha$ denote the quantity $\alpha_{R}$. Moreover, all the quantities referred to a distance are measured in $R_{0}$-units. It is easy to see that

$$
\begin{aligned}
& \Phi^{(a)}(0)=B \Phi^{(r)}(0), \quad B=2 \gamma^{3} e^{(1-\gamma) / \alpha}, \\
& \Psi(0)=A_{\gamma} \Phi^{(r)}(0), \quad A_{\gamma}=A e^{\left(n_{0}-\gamma\right) / \alpha}\left(\gamma / n_{0}\right)^{3} .
\end{aligned}
$$

For values (5) of the parameters $R_{0}$ and $\alpha$, the quantity $B$ expressed in (11) is small for the large values of $\gamma(\gamma \gg 1)$. Moreover, $B \geq 1$, when $\gamma \leq \gamma_{0}$, where $\gamma_{0}=1.87047$. The behavior of the coefficient $B(\gamma)$ is important in the subsequent calculations, in particular, when determining the relation between the Fourier transforms of the repulsive $\Phi^{(r)}(0)$ and attractive $\Phi^{(a)}(0)$ interactions. In the range $\gamma<\gamma_{0}$, we have

$\Phi^{(a)}(0)>\Phi^{(r)}(0)$.

This condition allows us to take advantage of the results of [12], where we obtained the following expression for the grand partition function of the cell fluid model:

$\Xi=\int(d \rho)^{N_{v}} \exp \left[\sqrt{N_{v}} \beta \tilde{\mu} \rho_{0}+\frac{\beta}{2} \sum_{\mathbf{k} \in B_{\mathrm{c}}} W(k) \rho_{\mathbf{k}} \rho_{-\mathbf{k}}\right] \times$

$\times \prod_{l=1}^{N_{v}}\left(\sum_{m=0}^{\infty} \frac{\left(\alpha^{*}\right)}{m !} e^{-p m^{2}} e^{m \tilde{t}_{1}}\right)$.

Here, $\alpha^{*}=v e^{\beta_{\mathrm{c}} \mu^{*}}, \tilde{\mu}=\mu-\mu^{*}(1+\tau)$. The effective interaction potential in the case of a modified Morse potential is as follows:

$W(k)=\Phi^{(a)}(k)-\Phi^{(r)}(k)-\Psi(k)+$

$+\frac{\beta_{\mathrm{c}}}{\beta} \chi_{0} \Phi^{(r)}(0)+\frac{\beta_{\mathrm{c}}}{\beta} \Psi(0)$.

Here, $\chi_{0}, \mu^{*}$ are some constants, and $\tau$ is the relative temperature,

$\tau=\left(T-T_{\mathrm{c}}\right) / T_{\mathrm{c}}$.

Here, $T_{\mathrm{c}}$ is the critical temperature which will be defined later on. It is easy to see that

$W(0)=\Phi^{(r)}(0)\left[B-1+\chi_{0}+\tau\left(\chi_{0}+A_{\gamma}\right)\right]$.

The condition $W(0)>0$ is met for all $\gamma<\gamma_{0}$. The parameter $p$ from (12) has the form

$p=\frac{\beta_{\mathrm{c}}}{2} \Phi^{(r)}(0)\left[\chi_{0}+A_{\gamma}\right]$.
Expressing the last factor in (12) as a cumulant series gives [12]

$$
\begin{aligned}
& \Xi=g_{v} \int(d t)^{N_{v}} \exp \left[-\frac{1}{2} \sum_{\mathbf{k} \in B_{\mathbf{c}}} t_{\mathbf{k}} t_{-\mathbf{k}} / \beta W(k)\right] \times \\
& \times \prod_{l=1}^{N_{v}} \exp \left(\sum_{m=0}^{\infty} \frac{g_{n}}{n !} \tilde{t}_{\mathbf{l}}^{n}\right) .
\end{aligned}
$$

In the last expression, the Stratonovich-Hubbard transform has been already used for the factor in (12) containing the effective interaction potential, since $W(k)>0$, as follows with (13). The quantity $g_{v}$ in (17) is given by the formula

$g_{v}=\prod_{\mathbf{k} \in B_{\mathbf{c}}}(2 \pi \beta W(k))^{-1 / 2}$,

and the variable $\tilde{t}_{\mathbf{k}}$ is

$\tilde{t}_{\mathbf{k}}=t_{\mathbf{k}}+\beta_{\mathrm{c}} \tilde{\mu} \sqrt{N_{v}} \delta_{\mathbf{k}}$

Moreover,

$\tilde{t}_{\mathbf{l}}=\frac{1}{\sqrt{N_{v}}} \sum_{\mathbf{k} \in B_{\mathrm{c}}} \tilde{t}_{\mathbf{k}} e^{-i \mathbf{k} \mathbf{l}}$.

The coefficients $g_{n}$ from $[8,12]$ are as follows:

$g_{0}=\ln T_{0}, \quad g_{1}=T_{1} / T_{0}, \quad g_{2}=T_{2} / T_{0}-g_{1}^{2}$,

$g_{3}=T_{3} / T_{0}-g_{1}^{3}-3 g_{1} g_{2}$,

$g_{4}=T_{4} / T_{0}-g_{1}^{4}-6 g_{1}^{2} g_{2}-4 g_{1} g_{3}-3 g_{2}^{2}$,

where $T_{n}\left(p, \alpha^{*}\right)$ are the following special functions:

$T_{n}\left(p, \alpha^{*}\right)=\sum_{m=0}^{\infty} \frac{\left(\alpha^{*}\right)^{m}}{m !} m^{n} e^{-p m^{2}}$.

Since $p$ fails to be a function of the temperature, the coefficients of the Jacobian of the transition to collective variables $g_{n}$ do not depend on $\tau$.

In the approximation of the $\rho^{4}$ model, we have [12]

$$
\begin{aligned}
& \Xi=g_{v} e^{N_{v} E_{\mu}} \int(d \rho)^{N_{v}} \times \\
& \times \exp \left[M N_{v}^{1 / 2} \rho_{0}+\frac{1}{2} \sum_{\mathbf{k} \in \mathcal{B}_{\mathrm{c}}} \tilde{D}(k) \rho_{\mathbf{k}} \rho_{-\mathbf{k}}+\right. \\
& \left.+\frac{g_{4}}{24} \frac{1}{N_{v}} \sum_{\substack{\mathbf{k}_{1}, \ldots, \mathbf{k}_{4} \\
\mathbf{k}_{i} \in \mathcal{B}_{\mathrm{c}}}} \rho_{\mathbf{k}_{1}} \ldots \rho_{\mathbf{k}_{4}} \delta_{\mathbf{k}_{1}+\ldots+\mathbf{k}_{4}}\right]
\end{aligned}
$$

ISSN 2071-0194. Ukr. J. Phys. 2020. Vol. 65, No. 5 
The following notations are used in the last formula:

$$
\begin{aligned}
& E_{\mu}=g_{0}-\frac{\beta \tilde{\mu}^{2}}{2 W(0)}+n_{\mathrm{c}}\left(g_{1}+\frac{\tilde{\mu}}{W(0)}\right)+\frac{n_{\mathrm{c}}^{2}}{2} \tilde{D}(0)+\frac{g_{3}^{4}}{8 g_{4}^{3}}, \\
& M=\tilde{\mu} / W(0)+g_{1}+n_{\mathrm{c}} \tilde{D}(0)-\frac{1}{6} \frac{g_{3}^{3}}{g_{4}^{2}}, \\
& \tilde{D}(k)=\tilde{g}_{2}-1 / \beta W(k), \\
& \tilde{g}_{2}=g_{2}-\frac{1}{2} \frac{g_{3}^{2}}{g_{4}}, \quad n_{\mathrm{c}}=-g_{3} / g_{4} .
\end{aligned}
$$

Expression (21) is approximated only in the sense that it involves the contribution from the cumulants $g_{n}$ of $n \leq 4$. The higher $g_{n}$ (with $n \geq 5$ ) are considered to be zero. As shown in [15], this approximation qualitatively describes the behavior of a three-dimensional Ising model near the critical point. Since the simple fluids belong to the universality class of the Ising model, hopefully, the approximation used in (21) will allow us to describe the firstorder phase transition in simple fluids, including the critical region.

\section{Temperature Dependence and the Range of Model Parameters}

To compute the values of $g_{n}$ that are required to obtain the explicit form of (21), it is essential to have the parameters $p$ and $\alpha^{*}$. Consider the easiest case where

$p=1.0, \quad \alpha^{*}=5.0$.

The value of $p$ is unambiguously determined by the parameters $\chi_{0}$ and $A_{\gamma}$, which are a part of (16). The value of $A_{\gamma}$ is a function of the parameter $n_{0}$ defined in (11), so the case of $p=1$ corresponds to the welldefined $\chi_{0}$ and $n_{0}$. Let us find their magnitudes. According to (11), if

$\gamma=1.650$

then

$n_{0}=1.521, \quad A_{\gamma}=0.201 ; \quad \chi_{0}=0.070$.

We now examine (21) applying the zero-mode approximation, just as it was done in [12]. The result is a grand partition function in the following form:

$\Xi=g_{v} \exp \left[N_{v}\left(E_{\mu}+E\left(\bar{\rho}_{0}\right)\right)\right]$, where

$E\left(\bar{\rho}_{0}\right)=M \bar{\rho}_{0}+\frac{1}{2} \tilde{D}(0) \bar{\rho}_{0}^{2}-\frac{a_{4}}{24} \bar{\rho}_{0}^{4}$,

and the coefficient $a_{4}=-g_{4}>0$. The quantity $\bar{\rho}_{0}$ is a solution of the equation $\partial E\left(\rho_{0}\right) / \partial \rho_{0}=0$, which is

$\bar{M}+\tilde{D}(0) \bar{\rho}_{0}-\frac{a_{4}}{6} \bar{\rho}_{0}^{3}=0$.

Here, $\bar{M}$ is some value of the chemical potential which corresponds to an extremum of the function $E\left(\rho_{0}\right)$. This extremum is the maximum (which is a condition of the Laplace method used to calculate (25)), if the inequality

$\frac{\partial^{2} E\left(\bar{\rho}_{0}\right)}{\partial \rho_{0}^{2}} \leq 0$

is fulfilled. The equality condition in (28) corresponds to the quantity

$\rho_{0 r}^{2}=2 \frac{\tilde{D}(0)}{a_{4}}$.

Later on, we will see that $\rho_{0 r}$ describes a spinodal curve that is specified only in range $T<T_{\mathrm{c}}$.

The critical temperature $T_{\mathrm{c}}$ is provided by solving the equation

$\tilde{D}\left(0, T_{\mathrm{c}}\right)=0$.

For the parameters given by (23) and (24), we have

$k_{\mathrm{B}} T_{\mathrm{c}}=\tilde{g}_{2} W\left(0, T_{\mathrm{c}}\right)=\tilde{g}_{2}\left(B-1+\chi_{0}\right) \Phi^{(r)}(0)$,

$k_{\mathrm{B}} T_{\mathrm{c}} \approx 4.995 D$.

We now find the explicit form of the dependence of $\tilde{D}(0)$ on the temperature, using (22). In view of (15), we have

$\frac{1}{\beta W(0)}=\tilde{g}_{2} \gamma_{\tau}, \quad \gamma_{\tau}=\frac{1+\tau}{1+\omega_{0} \tau}$,

where $\tilde{g}_{2}$ is a constant which is independent of the temperature and defined by the microscopic parameters (5) and (23), and

$\omega_{0}=\frac{\chi_{0}+A_{\gamma}}{B-1+\chi_{0}}$.

Formula (22) yields the following expression:

$\tilde{D}(0)=-\tau \frac{1-\omega_{0}}{1+\omega_{0} \tau} \tilde{g}_{2}$ 
The computation of the coefficients $g_{n}$ given by (19) implies that, under conditions (23), the value $\tilde{g}_{2}<1 / 2$. Therefore, $\omega_{0}=2 \tilde{g}_{2}<1$. Consequently, the quantity $\tilde{D}(0) \leq 0$ for all $\tau \geq 0$, and $\tilde{D}(0) \geq 0$ in the case $\tau \leq 0$. This leads us to the conclusion that, in the temperature range $\tau>0$, Eq. (29) has no real solutions for $\rho_{0 r}$. Therefore, there are no restrictions on the quantity $\bar{\rho}_{0}$ as a solution of Eq. (27). However, in the case $\tau<0$, there is a real quantity

$\rho_{0 r}=\left(2 \frac{\tilde{D}(0)}{a_{4}}\right)^{1 / 2}$,

as well as the following restriction on the value of $\bar{\rho}_{0}$ :

$\left|\bar{\rho}_{0}\right| \geq \rho_{0 r}$.

The equality sign in (36) matches a spinodal curve that limits the stability region of the system.

The reduced form of Eq. (27) is given by

$\bar{\rho}_{0}^{3}+p_{t} \bar{\rho}_{0}+q=0$,

where

$p_{t}=-\frac{6 \tilde{D}(0)}{a_{4}} ; \quad q=-\frac{6 \bar{M}}{a_{4}}$.

The solution of (37) in the range $T>T_{\mathrm{c}}$ is given by

$\bar{\rho}_{0}=\left(\frac{3 \bar{M}}{a_{4}}+\sqrt{Q_{t}}\right)^{1 / 3}-\left(-\frac{3 \bar{M}}{a_{4}}+\sqrt{Q_{t}}\right)^{1 / 3}$,

where

$Q_{t}=\left(-\frac{2 \tilde{D}(0)}{a_{4}}\right)^{3}+\left(\frac{3 \bar{M}}{a_{4}}\right)^{2}$.

For all $T>T_{\mathrm{c}}$, the quantity $p_{t}>0$. This suggests the existence of a unique real solution of (37). Using both a well-known relation

$P V=k_{\mathrm{B}} T \ln \Xi$

and expression (25) gives the following explicit form of the equation of state in the case $T>T_{\mathrm{c}}$ :

$\frac{P v}{k_{\mathrm{B}} T}=\frac{1}{N_{v}} \ln g_{v}+E_{\mu}(T)+\bar{M} \bar{\rho}_{0}+\frac{1}{2} \tilde{D}(0) \bar{\rho}_{0}^{2}-\frac{a_{4}}{24} \bar{\rho}_{0}^{4}$.

The quantity $E_{\mu}(T)$ takes the form

$E_{\mu}(T)=g_{0}-\frac{\beta W(0)}{2}\left(\frac{\tilde{\mu}}{W(0)}\right)^{2}+\bar{M} n_{\mathrm{c}}-\frac{n_{\mathrm{c}}^{2}}{2} \tilde{D}(0)-\frac{1}{24} \frac{g_{3}^{4}}{g_{4}^{3}}$
$\frac{\tilde{\mu}}{W(0)}=\bar{M}-g_{1}-n_{\mathrm{c}} \tilde{D}(0)+\frac{1}{6} g_{3}^{3} / g_{4}^{2}$,

Note that, for all $\tau>0$, there are no restrictions on the value of $\bar{\rho}_{0}$. Expression (42) for the pressure is a monotonically increasing function of the chemical potential and the temperature.

\section{Region of Temperatures Below the Critical One}

For all $\tau<0$, Eq. (37) has three real solutions:

$\rho_{01}=2 \rho_{\text {or }} \cos \left(\alpha_{t} / 3\right)$,

$\rho_{02}=-2 \rho_{\text {or }} \cos \left(\alpha_{t} / 3+\frac{\pi}{3}\right)$,

$\rho_{03}=-2 \rho_{\text {or }} \cos \left(\alpha_{t} / 3-\frac{\pi}{3}\right)$,

where $\rho_{0 r}$ is given by (35). The quantity $\alpha_{t}$ is provided by

$\cos \alpha_{t}=\bar{M} / M_{q}$.

Moreover,

$M_{q}=-\frac{g_{4}}{3} \rho_{0 r}^{3}=\frac{a_{4}}{3}\left(\frac{2 \tilde{D}(0)}{a_{4}}\right)^{3 / 2}$.

Note that solutions (44) are valid in the case of $Q_{t}<0$, where $Q_{t}$ is defined in (40), that is, for all $|\bar{M}|<M_{q}$, where $M_{q}$ is a solution of the equation $Q_{t}=0$. The unique real solution $\bar{\rho}_{0}$ of Eq. (37) exists in the range $|M| \geq M_{q}$. It is calculated similarly to (39), because the quantity $Q_{t} \geq 0$ for these values of $M$ :

$\rho_{04}=\left(\frac{3}{a_{4}}\right)^{1 / 3}\left(A_{1}^{1 / 3}+A_{2}^{1 / 3}\right)$.

Here,

$A_{1}=\bar{M}+\sqrt{\bar{M}^{2}-M_{q}^{2}}$,
$A_{2}=\bar{M}-\sqrt{\bar{M}^{2}-M_{q}^{2}}$.

The solution $\rho_{04}$ defined in (47) is an extension of one of solutions (44). Moreover, for all $\bar{M}>0 . \rho_{04}$ is a continuation of the solution $\rho_{01}$. In the region $\bar{M}<0$, it coincides with $\rho_{03}$ at $\bar{M}=-M_{q}$. The corresponding curves are shown in Fig. 1.

Note that

$\lim _{\bar{M} \rightarrow M_{q}} \rho_{01}=\lim _{\bar{M} \rightarrow M_{q}} \rho_{04}^{(p)}=2 \rho_{0 r}$,

$\lim _{\bar{M} \rightarrow-M_{q}} \rho_{03}=\lim _{\bar{M} \rightarrow-M_{q}} \rho_{04}^{(m)}=-2 \rho_{0 r}$,

ISSN 2071-0194. Ukr. J. Phys. 2020. Vol. 65, No. 5 
where $\rho_{04}^{(p)}$ and $\rho_{04}^{(m)}$ correspond to (47) in either the region $\bar{M}>0$ or $\bar{M}<0$, respectively.

The equation of state at $T<T_{\mathrm{c}}$ in terms of the chemical potential and temperature has the following form:

$\frac{P v}{k_{B} T}=\frac{1}{N_{v}} \ln g_{V}+E_{\mu}(\mu, T)+E\left(\rho_{01}\right) \times$

$\times \Theta(M) \Theta\left(M_{q}-M\right)+E\left(\rho_{03}\right) \Theta(-M) \Theta\left(M_{q}+M\right)+$

$+E\left(\rho_{04}^{(m)}\right) \Theta\left(-M-M_{q}\right)+E\left(\rho_{04}^{p}\right) \Theta\left(M-M_{q}\right)$,

where the function $E\left(\rho_{0}\right)$ is specified in (26), and the arguments of this function are expressed by (44) and (47). The quantity $E_{\mu}(\mu, T)$ is given by (43). The 3D plot of the pressure below $T_{\mathrm{c}}$ is represented in Fig. 2 .

\section{Relation between the Chemical Potential and the Density}

The above-calculated equations of state (42) at $T>T_{\mathrm{c}}$ and (50) at $T<T_{\mathrm{c}}$ contain the dependence of the pressure on the temperature and the chemical potential. To obtain the pressure as a function of the temperature and density, we use the known relation for the average number of particles:

$\langle N\rangle=\frac{\partial}{\partial \beta \mu} \ln \Xi$

Taking expression (25) into account, we have

$\bar{n}=\frac{\langle N\rangle}{N_{v}}=\frac{\partial E_{\mu}}{\partial \beta \mu}+\frac{\partial E\left(\bar{\rho}_{0}\right)}{\partial \beta \bar{M}}$.

The following equation which links the density of particles $\bar{n}$ and the chemical potential $\bar{M}$ is obtained using formulas (26) and (43):

$\bar{n}=n_{g}-\bar{M}+\tilde{g}_{2} \gamma_{\tau} \bar{\rho}_{0}$,

where

$n_{g}=g_{1}+n_{\mathrm{c}} \tilde{g}_{2}-\frac{1}{6} g_{3}^{3} / g_{4}^{2}$.

Rewrite (53) in the form

$\bar{\rho}_{0}=\left(\bar{M}+\bar{n}-n_{g}\right) / \tilde{g}_{2} \gamma_{\tau}$.

This expression is actually the equation for $\rho_{0}$, since $\bar{M}$ contained in (53) is a function of the quantity $\bar{\rho}_{0}$. This follows from theequality (27), which specifies the extreme value of $\bar{\rho}_{0}(\tau, \bar{M})$. Substituting

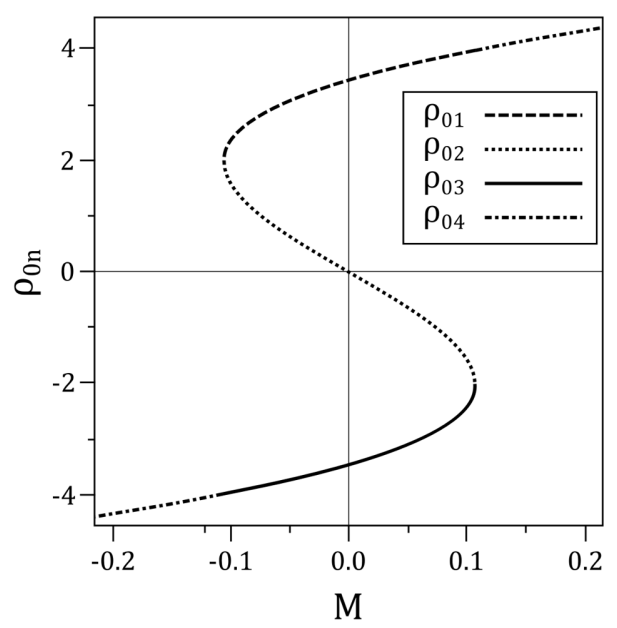

Fig. 1. Plot of solutions $\bar{\rho}_{0}$ (see (44)) at $\tau=-0.5$. There exist three real roots for $Q_{t}<0$ and a unique real solution for $Q_{t}>0$

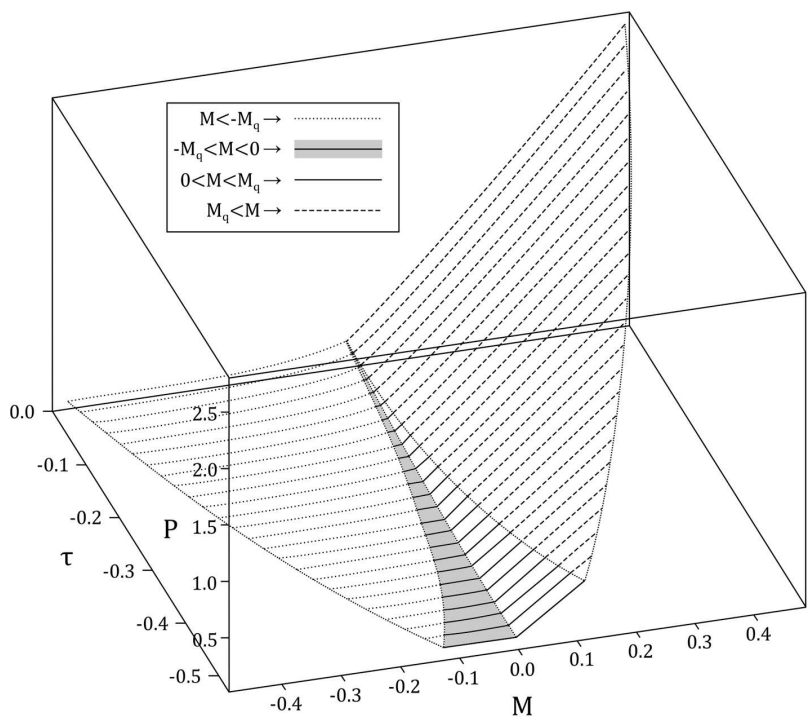

Fig. 2. 3D plot of the pressure as a function of the temperature and the chemical potential $(P=P(T, M))$ in the region $T<T_{\mathrm{C}}$

$\bar{M}$ from (27) to (55), we obtain the equation for $\rho_{0 n}=\rho_{0 n}(\tau, \bar{n})$ :

$\rho_{0 n}^{3}+p_{q} \rho_{0 n}+q_{q}=0$.

Here, the coefficients $p_{q}$ and $q_{q}$ are given by

$p_{q}=-\frac{6}{a_{4}}\left(\tilde{g}_{2} \gamma_{\tau}+\tilde{D}(0)\right) ; \quad q_{q}=\frac{6}{a_{4}}\left(\bar{n}-n_{g}\right)$.

Equation (56) allows us to find $\bar{\rho}_{0}$ as a function of the density $\bar{n}$ and the temperature. It transforms the 


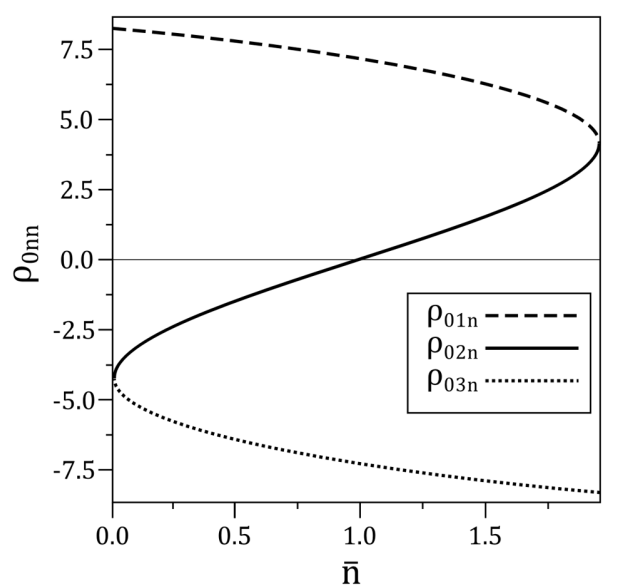

Fig. 3. Plot of the solutions $\rho_{0 n n}$ given by (60) as functions of the density

dependence of the solution of Eq. (37) on chemical potential to that on the density.

It is easy to see that, in view of (32) and (34), the coefficient $p_{q}$ is a constant. We have

$p_{q}=-\frac{6 \tilde{g}_{2}}{a_{4}}$.

It is negative and independent of the temperature. Therefore, for all $Q_{q}<0$ given by

$Q_{q}=\left(p_{q} / 3\right)^{3}+\left(q_{q} / 2\right)^{2}$,

Eq. (56) possesses three real solutions:

$$
\begin{aligned}
\rho_{01 n} & =2\left(\frac{2 \tilde{g}_{2}}{a_{4}}\right)^{1 / 2} \cos \left(\alpha_{n} / 3\right), \\
\rho_{02 n} & =-2\left(\frac{2 \tilde{g}_{2}}{a_{4}}\right)^{1 / 2} \cos \left(\alpha_{n} / 3+\pi / 3\right), \\
\rho_{03 n} & =-2\left(\frac{2 \tilde{g}_{2}}{a_{4}}\right)^{1 / 2} \cos \left(\alpha_{n} / 3-\pi / 3\right) .
\end{aligned}
$$

Here, either

$\cos \alpha_{n}=\frac{n_{g}-\bar{n}}{n_{\varphi}} ; \quad n_{\varphi}=\frac{2}{3}\left(\frac{2 \tilde{g}_{2}^{3}}{a_{4}}\right)^{1 / 2}$

or

$\alpha_{n}=\arccos \left(\frac{n_{g}-\bar{n}}{n_{\varphi}}\right)$.

Solutions (60) are shown in Fig. 3. The extension of (56) to the region $Q_{q}>0$ leads to the continuation of non-physical branches of the solutions $\rho_{03 n}$ and $\rho_{01 n}$ (see Fig. 3).

434
Note that both (37) and (56) are equations of the same type, but describe different dependences. We can find the solutions $\bar{\rho}_{0}(\tau, M)$ of $(37)$ to determine the extrema of $E\left(\bar{\rho}_{0}\right)$ provided by $(26)$, by applying the Laplace method [16] to calculate the grand partition function $\Xi(\tau, M)$. This is the way to obtain the equation of state (41) in terms of the temperature and chemical potential. Equation (56) allows us to find both the quantity $\bar{\rho}_{0}$ and, consequently, the pressure as functions of the density and temperature.

The difference between Eqs. (37) and (56) is also evident in the aspect of mathematics. Thus, the solutions $\rho_{01}$ and $\rho_{03}$ of Eq. (37) corresponding to $\max E\left(\bar{\rho}_{0}\right)$ are useful at $T<T_{\mathrm{c}}$. The quantity $\rho_{02}$ refers to $\min E\left(\bar{\rho}_{0}\right)$. Among the three solutions (56), only $\rho_{02 n}$ is physical. This solution shows that the density increases with the chemical potential. The other two solutions, $\rho_{01 n}$ and $\rho_{03 n}$, show a decrease in the density (with growing chemical potential). Such a behavior does not fit the reality. The calculations should be carried out in the sequence suggested in the present paper. First, we find the solutions $\bar{\rho}_{0}(\tau, M)$ which give an explicit expression of the grand partition function $\Xi(\tau, M)$ as a function of the temperature and chemical potential. Only then, we search for $\bar{\rho}_{0}(\tau, \bar{n})$. This procedure gives the way to obtain both $\Xi(\tau, \bar{n})$ and the equation of state $P=P(\tau, \bar{n})$ in terms of the temperature and density.

In the zero-mode approximation, Eq. (25) of the $\rho^{4}$-model (21) leads to a restriction on the quantity $\rho_{0 n}(\tau, \bar{n})$ defined in (60) (see Fig. 3):

$-\bar{\rho}<\rho_{0 n}<\bar{\rho}$,

where

$\bar{\rho}=\left(2 \tilde{g}_{2} / a_{4}\right)^{1 / 2}$.

Consider this situation in more details. For this purpose, we rewrite Eq. (53) as

$\bar{M}=\tilde{g}_{2} \gamma_{\tau} \rho_{0 n}-\left(\bar{n}-n_{g}\right)$.

The quantity $\bar{M}$ [which is a variable in the framework of the grand canonical ensemble in terms of $(\tau, M)$ ] becomes a function of the density $\bar{n}$ and the temperature $\tau$. To transform the results represented in terms of $(\tau, M)$ to those in terms of $(\tau, \bar{n})$, we use formula (64), where the solution $\rho_{02 n}$ from (60) is applied to $\rho_{0 n}$. To simplify notations, we denote $\rho_{02 n}=\rho_{n}$. Since the right-hand side of (64) is a finite quantity, the quantity $\bar{M}$ is also limited ISSN 2071-0194. Ukr. J. Phys. 2020. Vol. 65, No. 5 
by two boundary curves. The former corresponds to $\rho_{n}=-\bar{\rho}$. Then (64) gives

$M^{*}=\lim _{\rho_{n} \rightarrow-\bar{\rho}} \bar{M}=-\tilde{g}_{2} \gamma_{\tau} \bar{\rho}-\left(n_{\min }-n_{g}\right)$.

The following relation is used in the last formula:

$\lim _{\rho_{n} \rightarrow-\bar{\rho}} \bar{n}=n_{\min }$.

The other limit provides an upper boundary curve, where $\rho_{n} \rightarrow \bar{\rho}$, and the density $\bar{n} \rightarrow n_{\text {max }}$ :

$M^{* *}=\lim _{\rho_{n} \rightarrow \bar{\rho}} \bar{M}=\tilde{g}_{2} \gamma_{\tau} \bar{\rho}-\left(n_{\max }-n_{g}\right)$.

Here,

$\lim _{\rho_{n} \rightarrow \bar{\rho}} \bar{n}=n_{\max }$.

Figure 4 illustrates the temperature dependences of $M^{*}$ and $M^{* *}$. Evidently, there is no gas-liquid phase transition below the value $n_{\min }$ which specifies the lowest possible density for some temperature $T^{*}$. Thus, in the approximation of the $\rho^{4}$ distribution, the cell fluid model has a certain region of admissible density values:

$n_{\min } \leq \bar{n} \leq n_{\max }$.

It is easy to see that there is some temperature

$\tau^{*}=-0.618$,

at which the following equality is fulfilled:

$M^{*}=M^{* *}=0$.

This temperature corresponds to some fixed density values

$n_{\max }=n_{g}+n_{\varphi}$

$n_{\min }=n_{g}-n_{\varphi}$,

where

$n_{\varphi}=\bar{\rho} \tilde{g}_{2} \gamma_{\tau}\left(\tau^{*}\right)$.

Clearly, in view of the condition $n_{\min } \geq 0$, the following inequality should be held:

$n_{g} \geq n_{\varphi}$

This condition is met for the parameter set (23). We use parameters (24) to describe sodium and find

$n_{g}=0.977 ; \quad n_{\max }=1.946 ;$

$n_{\varphi}=0.968 ; \quad n_{\min }=0.009$.

ISSN 2071-0194. Ukr. J. Phys. 2020. Vol. 65, No. 5

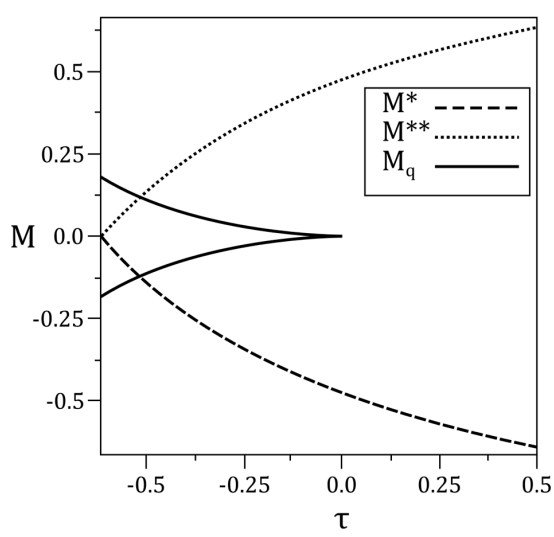

Fig. 4. Boundary values of the chemical potential $M\left(M_{q}\right.$, $M^{* *}$ - the upper limit, $M^{*}-$ the lower limit)

The quantity $n_{\min }$ increases, as the parameter $\alpha^{*}$ decreases. For instance, the equalities $p=1$ and $\alpha^{*}=4$ give

$n_{g}=0.884 ; \quad n_{\max }=1.652$;

$n_{\varphi}=0.767 ; \quad n_{\min }=0.117$.

In the case $p=1, \alpha^{*}=3$, and

$n_{g}=0.832 ; \quad n_{\max }=1.505$;

$n_{\varphi}=0.673 ; \quad n_{\min }=0.158$.

We note that each set of parameters $p$ and $\alpha^{*}$ corresponds to particular values of the characteristic densities.

At $T>T_{\mathrm{c}}$, the equation of state of the cell fluid model in terms of the density has the form

$\frac{P v}{k_{\mathrm{B}} T}=\frac{1}{N_{v}} \ln g_{v}+E_{\mu}^{(2)}(n, T)+\bar{M} \rho_{n}+$

$+\frac{\tilde{D}(0)}{2} \rho_{n}^{2}-\frac{a_{4}}{24} \rho_{n}^{4}$

where

$\bar{M}=\rho_{n} \tilde{g}_{2} \gamma_{\tau}-\left(\bar{n}-n_{g}\right)$

is a function of the density and temperature. We use formula (43) to find

$\frac{\tilde{\mu}}{W(0)}=\bar{M}-n_{g}+n_{\mathrm{c}} \tilde{g}_{2} \gamma_{\tau}$,

where $n_{g}$ is defined in (54). Therefore,

$E_{\mu}^{(2)}(n, T)=g_{0}-\frac{1}{2 \tilde{g}_{2} \gamma_{\tau}}\left(\frac{\tilde{\mu}}{W(0)}\right)^{2}+n_{\mathrm{c}} \bar{M}-$

$-\frac{1}{2} n_{\mathrm{c}}^{2} \tilde{D}(0)-\frac{1}{24} \frac{g_{3}^{4}}{g_{4}^{3}}$. 


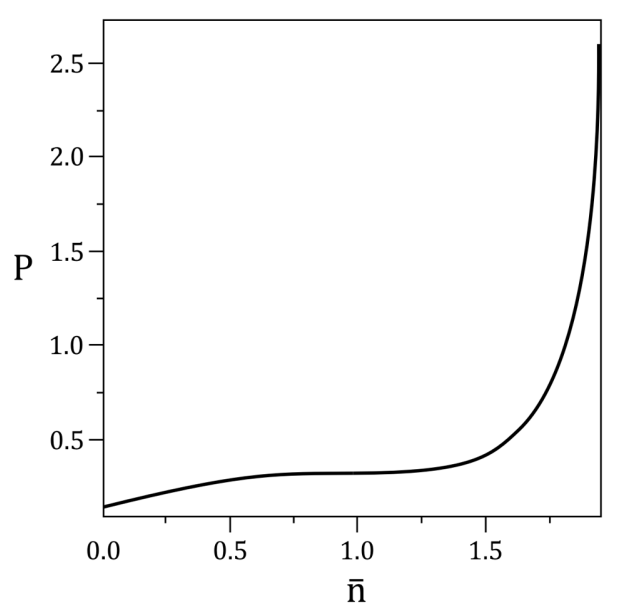

Fig. 5. Plot of the pressure as a function of the density at $T=T_{\mathrm{c}}$

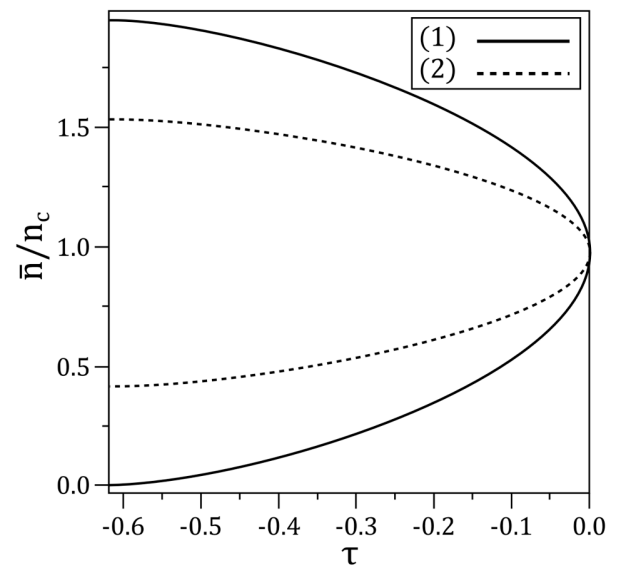

Fig. 6. Coexistence curve (1) and the spinodal (2) of the model investigated in the present research

The plot of the pressure as a function of the density at $T=T_{\mathrm{c}}$ is represented in Fig. 5 . It shows that $n_{g}$ is the critical density.

\section{Phase Behavior of the Cell Fluid Model}

In Sections 3 and 4 , the equation $P=P(\tau, M)$ is obtained for the temperature regions above and below the critical temperature. Section 5 states that the region of the chemical potential $M$ is limited by two characteristic curves $M^{*}(65)$ and $M^{* *}(67)$, respectively,

$M^{*} \leq M \leq M^{* *}$.

Figure 2 shows the $3 \mathrm{D}$ plot of the equation of state $P(\tau, M)$ for the temperatures $T<T_{\mathrm{c}}$. It has a frac- ture along with the line $M=0$, which indicates a first-order phase transition, since the first derivative of the pressure with respect to the chemical potential $M$ on the line $M=0$ will be different from the left- and right-hand sides. In addition, Fig. 4 shows the line $M_{q}(\tau)$, which delimits regions of the surface $P(\tau, M)$, in which there are either one or three real solutions of Eq. (37) with the unknown $\bar{\rho}_{0}$. Finding $\bar{\rho}_{0}$ makes it possible to calculate the grand partition function of the cell fluid model and to establish the existence of a first-order phase transition in it. Figure 6 illustrates the coexistence curve and the spinodal of the present model. Each of these curves ends at a temperature $T=T^{*}<T_{\mathrm{c}}$.

\section{Conclusions}

The results of the present research state that the cell fluid model is suitable for the description of a firstorder phase transition. The interaction potential of such a model, in addition to the attractive and repulsive interactions (such as the Morse potential), should include an additional repulsive interaction (in a reference system). The use of the supplementary interaction enables studying a phase behavior of the model in wide ranges of the density and the temperature.

A feature of the proposed approach is the use of the grand canonical ensemble, in which the temperature and the chemical potential are independent variables. First, the equation of state of the cell fluid model is obtained in terms of these variables and then transposed to the temperature-density plane. For this purpose, we derived and solved the equation, which relates the average number of particles (density) to the chemical potential. The solutions are functions of the temperature. Such dependence is found to be substantially nonlinear. Moreover, it implies the limited lower and upper ranges of the density and the chemical potential. We have proved that the minimum and maximum admissible values of the chemical potential approach each other below the critical temperature. The estimated value of the temperature $T^{*}$, at which they coincide, is $T^{*} \approx 0.38 T_{\mathrm{c}}$ (for the sodium-specific interaction potential parameters given in [14]). The conclusion on the existence of a first-order phase transition in the temperature region $T^{*}<T<T_{\mathrm{c}}$, as well as the absence of the liquid-gas phase transition in the temperature region $T \leq T^{*}$, is obvious. The temperature $T^{*}$ can be interpreted as the triple point temperature. We obtain both the 
maximum density value $n_{\max }$ for the liquid phase and the minimum density value in the gaseous phase $n_{\text {min }}$ (formula (68)). Each of these densities corresponds to the temperature specified in formula (66).

The proposed method for calculating the phase behavior can be extended to other systems with the known parameters of interaction potentials.

The equation obtained in this paper is not suitable near the critical point $\left(\tau<\tau^{*} \approx 10^{-2}\right)$. In this case, one should count the effects of long-range fluctuations, since they play a major role in the description of the critical region above and below the critical point. In this regard, there are theoretical approaches [4, 17-19] and experiments [20-22] confirming the presence of the non-classical critical exponents of compressibility, heat capacity, correlation length, etc. We have also considered fluctuations (within the approach represented in the present paper) based on the calculation of the grand thermodynamic potential and on the consideration of the contributions of the collective variables $\rho_{\mathbf{k}}$ with $|\mathbf{k}| \neq 0$ [11].

1. A.J. Schultz, D.A. Kofke. Vapor-phase metastability and condensation via the virial equation of state with extrapolated coefficients. Fluid Phase Equilibria 409, 12 (2016).

2. A.J. Masters. Virial expansions. J. Phys.: Condens. Matter 20, 283102 (2008).

3. M.V. Ushcats. Modified Lennard-Jones model: Virial coefficients to the 7th order. J. Chem. Phys. 140, 234309 (2014).

4. D. Pini, G. Stell, N.B. Wilding. A liquid-state theory that remains successful in the critical region. Mol. Phys. 95, 483 (1998).

5. C.-L. Lee, G. Stell, J. Hoye. A simple SCOZA for simple fluids. J. Mol. Liq. 112, 13 (2004).

6. Y. Kozitsky, M. Kozlovskii, O. Dobush. Phase transitions in a continuum Curie-Weiss system: A quantitative analysis. In: Modern Problems of Molecular Physics (Springer, 2018), pp. 229-251.

7. M.P. Kozlovskii, O.A. Dobush. Phase transition in a cell fluid model. Condens. Matter Phys. 20, 23501 (2017).

8. M.P. Kozlovskii, O.A. Dobush, I.V. Pylyuk. Using a fluid cell model for description of a phase transition in simple liquid alkali metals. Ukr. J. Phys. 62, 865 (2017).

9. I. Yukhnovskii, V. Kolomiets, I. Idzyk. Liquid-gas phase transition at and below the critical point. Condens. Matter Phys. 16, 23604 (2013).

10. I.R. Yukhnovskii. The phase transition of the first order in the critical region of the gas-liquid system. Condens. Matter Phys. 17, 43001 (2014).

11. M.P. Kozlovskii, I.V. Pylyuk, O.A. Dobush. The equation of state of a cell fluid model in the supercritical region. Condens. Matter Phys. 21, 43502 (2018).
12. M. Kozlovskii, O. Dobush. Representation of the grand partition function of the cell model: The state equation in the mean-field approximation. J. Mol. Liq. 215, 58 (2016).

13. R.C. Lincoln, K.M. Koliwad. Morse-potential evaluation of second- and third-order elastic constants of some cubic metals. Phys. Rev. 157, 463 (1967).

14. J.K. Singh, J. Adhikari, S.K. Kwak. Vapor-liquid phase coexistence curves for Morse fluids. Fluid Phase Equilibria 248, 1 (2006).

15. I.R. Yukhnovskii, M.P. Kozlovskii, I.V. Pylyuk. Thermodynamics of three-dimensional Ising-like systems in the higher non-Gaussian approximation: Calculational method and dependence on microscopic parameters. Phys. Rev. B 66, 134410 (2002).

16. M.V. Fedoryuk. Asymptotic methods in analysis. In: Analysis I: Integral Representations and Asymptotic Methods. Edited by M.A. Evgrafov, R.V. Gamkrelidze (Springer, 1989).

17. A. Parola, L. Reatto. Recent developments of the hierarchical reference theory of fluids and its relation to the renormalization group. Mol. Phys. 110, 2859 (2012).

18. J.-M. Caillol. Non-perturbative renormalization group for simple fluids. Mol. Phys. 104, 1931 (2006).

19. I.R. Yukhnovskii. Phase space of collective variables and the Zubarev transition function. Theor. Math. Phys. 194, 224 (2018).

20. A. Oleinikova, L. Bulavin, V. Pipich. Critical anomaly of shear viscosity in a mixture with an ionic impurity. Chem. Phys. Let. 278, 121 (1997).

21. G. Brunner. Near critical and supercritical water. Part I. Hydrolytic and hydrothermal processes. J. Supercrit. Fluids 47, 373 (2009).

22. G. Brunner. Near and supercritical water. Part II: Oxidative processes. J. Supercrit. Fluids 47, 382 (2009).

Received 19.11.19

\section{М.П. Козловсъкий, О.А. Добуш}

\section{ФАЗОВА ПОВЕДІНКА МОДЕЛІ КОМІРКОВОГО}

\section{ПЛИНУ З МОДИФІКОВАНИМ ПОТЕНЦІАЛОМ МОРЗЕ}

$\mathrm{P}$ е $з$ ю м е

$\mathrm{У}$ роботі здійснено теоретичний опис фазового переходу першого роду коміркової моделі плину із модифікованим потенціалом Морзе та додатковою відштовхувальною взаємодією. У формалізмі великого канонічного ансамблю розраховано рівняння стану системи у змінних хімпотенціалтемпература та густина-температура для широкого діапазону густини і температури. Досліджено поведінку хімічного потенціалу як функції температури та густини. Виявлено максимально і мінімально допустимі значення хімпотенціалу, які наближаються одне до одного зі зменшенням температури. Встановлено існування фазового переходу рідинагаз в обмеженій області температур нижче критичної $T_{\mathrm{c}}$. 